\title{
LOS PARTIDOS TRASNACIONALES EN ESPAÑA. EL CASO DE LOS PARTIDOS RUMANOS
}

\author{
THE TRANSNATIONAL PARTIES IN SPAIN. THE CASE OF THE \\ ROMANIAN PARTIES
}

\author{
Antonio Alaminos Chica, \\ Clemente Penalva Verdú \\ Universidad de Alicante, Alicante. España/Spain \\ alaminos@ua.es \\ clemente.penalva@ua.es
}

Recibido/Received: 29/05/2017

Modificado/Modified: 30/06/2017

Aceptado/Accepted: 27/09/2017

\section{RESUMEN}

Enmarcada en los estudios sobre la vertiente sociopolítica de la transnacionalidad, esta investigación analiza dos organizaciones políticas diseñadas exógenamente (promovidas y coordinadas desde un ámbito y espacio supralocal o supranacional), inspiradas por la existencia de residentes extranjeros en varios municipios españoles, en este caso de nacionalidad rumana. Los datos proceden de la investigación documental, análisis estadístico de candidaturas y resultados electorales, y de entrevistas en profundidad a representantes de dichos partidos. El trabajo concluye que la presencia de un colectivo elevado de residentes extranjeros no son, per se, indicadores de potencial electoral; a no ser que medien los elementos motivadores de movilización. El argumento electoral de compartir la misma nacionalidad de origen no parece suficiente, aun cuando el programa político intente ser trasversal, evitando posiciones ideológicas y recurriendo a elementos genéricos como son la cultura común y su defensa, y la posibilidad de ayudar a regenerar la política y la democracia en el país de origen. Existen contradicciones importantes como son el enfoque supralocal y nacional en elecciones de lógica local, o la desconfianza previa de los electores potenciales en partidos rumanos sobre la base de la experiencia en su país de origen.

\section{PALABRAS CLAVE}

Migrantes; rumanos; política transnacional; elecciones locales; nuevos partidos políticos.

\section{SUMARIO}

1. Introducción. 2. Fuentes de datos. 3. Los partidos rumanos en España: entre el transnacionalismo y la política local. 3.1. El Partido Independiente Rumano (PIR). 3.2. El Partido Ibérico de los Rumanos (PIRUM). 4. Conclusiones. Bibliografía.

\section{ABSTRACT}

Based on the studies on the socio-political aspect of transnationality, this research analyzes two exogenously designed political organizations (promoted and coordinated from a supralocal or supranational scope and space), inspired by the existence of foreign residents in several Spanish 
municipalities, in this case Romanian nationality. The data come from documental research, statistical analysis of candidacies and electoral results, and from in-depth interviews with representatives of these parties. The paper concludes that the presence of a large group of foreign residents is not, per se, indicators of electoral potential; unless the motivating elements of mobilization are mediated. The electoral argument of sharing the same nationality of origin does not seem enough, even if the political program tries to be transversal, avoiding ideological positions and appealing to generic elements such as common culture and its defense, and the possibility of helping to regenerate politics and democracy in the country of origin. There are important contradictions such as the supralocal and national approach in local logic elections, or the previous distrust of potential voters in Romanian parties based on experience in their country of origin.

\section{KEYWORDS}

Migrants; Romanians; transnational politics; local elections; new political parties.

\section{CONTENTS}

1. Introduction. 2. Source of the data. 3. Romanians political parties in Spain and the local politics. 3.1. The Independent Romanian Party (PIR). 3.2.2. The Iberian Party for Romanians. 4. Conclusions. References.

\section{INTRODUCCIÓN}

Un concepto especialmente ligado al fenómeno migratorio es el de transnacionalidad. El concepto de la transnacionalidad, no exento de debate en sus contenidos, parte de una posición común basada en las actividades iniciadas y sostenidas por actores no estatales, organizados en grupos o redes de personas a través de las fronteras (Vertovec, 2007). En la discusión sobre su definición son destacables los trabajos de varios autores que clarifican y concretan el concepto en sus dimensiones teóricas y metodológicas (Portes, Guarnizo y Landolt, 1999; Guarnizo, Portes y Haller, 2003). En nuestra investigación se considera que las redes y grupos transnacionales presentan las características de ser regulares, sostenibles y simultáneas (Vertovec, 2003; Guarizo, Portes y Haller, 2003; Levitt y Schiller, 2004) e involucran a un número significativo de migrantes y sus contrapartes en el país de origen. La forma en que estos grupos transnacionales se constituyen como tales es muy diversa, identificándose una amplia gama de actividades de orden económico, asociativo, cultural y político que se entrecruzan entre sí. En ese sentido, implican en mayor o menor medida a las poblaciones e instituciones tanto del país de origen como del de destino.

En general, las conexiones socioeconómicas y políticas que traspasan las fronteras de varios Estados son producidas principalmente por la migración interestatal (Schiller, Basch, Szanton-Blanc, 1992; Basch, Schiller, Szanton-Blanc, 1994; Portes, 1996; Portes, 2001; Smith y Guarnizo, 1998) y los movimientos sociales (Smith, Chatfield y Pagnucco, 1997; Tarrow, 1998) o las ONG (Keck y Sikkink, 1998; Boli y Thomas, 1999). En este contexto, la transnacionalidad emerge como un concepto particular, y el transnacionalismo se define como "los procesos a través de los cuales los inmigrantes construyen campos sociales que conectan su país de origen y su país de asentamiento. (...) Los transmigrantes desarrollan y mantienen múltiples relaciones -familiares, económicas, sociales, organizacionales, religiosas, políticas- que sobrepasan fronteras. Los transmigrantes actúan, toman decisiones y se sienten implicados, y desarrollan identidades dentro de redes sociales que les conectan a ellos con dos o más sociedades de forma simultánea" (Schiller, Basch, Szanton-Blanc, 1992: 1-2). 
Por otro lado, considerando sus efectos sociopolíticos, se ha de asumir, en primer lugar, que en un contexto de globalización y del incremento de la movilidad humana asociada, el transnacionalismo lleva consigo la generación de espacios políticos desterritorializados (Guarizo y Smith, 1999); y, en segundo lugar, que estos nuevos espacios y su posible consolidación ponen en cuestión la vigencia y futuro de los estados-nación tal como fueron concebidos por la modernidad. Si el campo social transnacional es un terreno ilimitado de redes egocéntricas entrelazadas que configuran los migrantes, incluyendo el estado de origen y el de residencia (Schiller y Fouron, 1999), resulta de gran relevancia observar sus derivaciones sociopolíticas. Las prácticas cívico-políticas que estas redes van desarrollando en diferentes territorios y estados contribuyen a redefinir nociones y ordenamientos legales que fueron concebidos durante las formaciones de los estados modernos, como son las bases de la ciudadanía y la nacionalidad, así como los derechos asociados a ella. En el caso de la Unión Europea, los ordenamientos jurídicos que regulan varios derechos políticos se han modificado para facilitar la inclusión progresiva de las personas migrantes al sistema político, tanto en garantías como en participación en los procesos electorales.

En este punto se sitúa el debate sobre si el mantenimiento de las redes asociativas con el lugar de origen y el no abandono de la lealtad a sus países de nacimiento entorpece la adquisición de la nacionalidad en el lugar de destino. Para varios autores la doble lealtad no retarda la incorporación política de los inmigrantes en EE. UU., sino que a partir de la participación en sus asociaciones se incrementa su interés en participar electoralmente en el país de destino: "En efecto, y contradiciendo los avisos de los asimilacionistas, parece ser perfectamente posible que los inmigrantes se hagan un hueco en el sistema norteamericano integrándose políticamente en él a la vez que mantienen sus intereses y lealtades a los países de los que vinieron" (Portes, Escobar, Arana, 2008: 59).

De forma paralela a esta discusión sobre el papel integrativo de la "doble lealtad"se sitúa, en términos electorales, la opción que confieren los sistemas políticos a la población inmigrante de conservar el derecho al voto en el país de origen tras migrar a otro país. Esto abre la posibilidad de participar a distancia en la decisión sobre los gobiernos y, con ello, una forma específica de hacer política. Algunos autores introducen la noción de ciudadanía transnacional como concepto que permite integrar las migraciones internacionales y la actuación política transnacional. Desde esta posición, Parra (2016) señala dos modelos jurídicos subyacentes en la participación política de la ciudadanía transnacional: el romano y el aristotélico, vinculados al lugar de origen y destino, respectivamente. Dos modelos que han servido para establecer una tipología de tipos de orientaciones sobre las cuales la opinión pública europea concibe la noción de ciudadanía en los casos de movilidad internacional (Alaminos, Penalva, Perea, 2017). Según Parra, la ciudadanía transnacional tendría como fundamento la conservación del derecho al sufragio en el país de origen, afianzando los vínculos que comparten los migrantes transnacionales con su origen. Asimismo, supone, por parte del Estado-nación, "el reconocimiento de la importancia de una ciudadanía transnacional que pertenece a una comunidad política sin residencia fija." (Parra 2016: 93). El voto en el exterior, más allá de un mecanismo de participación electoral, es observado desde la perspectiva transnacional como una forma de afianzar tal pertenencia, incluso "independientemente del deseo del migrante y sus descendientes de retornar al país de origen” (Lafleur, 2012).

Sobre la base de dicho potencial transnacional, surgirán en España unos partidos políticos orientados a la consolidación y activación de las capacidades electorales del migrante para influir en los resultados electorales de su país de origen. Con carácter 
general, en la formación de partidos políticos promovidos por población migrante en España se pueden observar dos modelos. Las iniciativas de carácter endógeno, promovidas desde los propios intereses locales de los residentes extranjeros y aquellas otras que responden a diseños de carácter exógeno, promovidas y coordinadas desde una lógica supralocal o transnacional. Un primer aspecto a considerar son las limitaciones que la ley impone a la formación de partidos formados por personas de nacionalidad extranjera. Así, en el caso español existe una restricción evidente a la constitución de partidos políticos exclusivamente formados por extranjeros, es decir, sin nacionalidad española. Esto es así, de acuerdo con la sentencia 48/2003 del Tribunal Constitucional (Sentencia 48/2003) sobre el recurso de inconstitucionalidad promovido por el Gobierno Vasco impugnando varios preceptos de la Ley Orgánica de Partidos Políticos por la supuesta infracción del contenido del derecho de asociación. La sentencia alega razones relacionadas con el principio de soberanía popular. Aunque se reserva el derecho de sufragio activo y pasivo de los residentes europeos, así como su libre afiliación a los partidos, los excluye de la capacidad de creación de partidos políticos por ser éstos unas asociaciones cualificadas por la relevancia constitucional de sus funciones: las de representar a la soberanía en su conjunto, una soberanía reservada únicamente a los españoles.

Esta sentencia fue causa, como veremos, de la mutación acelerada de un partido político como el Partido Independiente Rumano (PIR), que se reinventaría como la Unión Demócrata Social (de Castellón). Aunque esa limitación no alcanza a la formación de agrupaciones de electores con presencia en las elecciones locales, no pueden existir partidos políticos formados exclusivamente por extranjeros porque deben de contar con la presencia de españoles. La Unión Europea ha sido el espacio ideal para la formulación y funcionamiento de auténticos partidos transnacionales, que transciendan las coaliciones o agrupación de partidos de referente nacional. Desde el marco regulatorio de la UE, existe una definición legal muy concreta: "Un partido político europeo (formalmente partido político a escala europea), es un peculiar tipo de organización política transnacional que opera al nivel de la Unión Europea (...) puede tratarse de dos formas de organización y participación política enteramente distintas, a saber: a) un partido político (stricto sensu), como asociación de ciudadanos de la Unión que persigue objetivos políticos y que esté reconocida como tal por el ordenamiento jurídico de al menos un Estado miembro; b) una coalición de partidos políticos, como cooperación estructurada, unidos con base en su afinidad ideológica con fines políticos" (Reglamento CE 2004/2003).

Sin embargo, la noción de transnacional excede la definición formal de la norma europea, y se extiende a la posibilidad de que ciudadanos emigrados influyan en las elecciones de su país de origen. Este es el caso que se presenta a continuación, el de los partidos de migrantes rumanos, donde la dimensión paneuropea parte de una estrategia de finalidad nacional (de origen) más que de una dimensión políticamente vertebradora entre diferentes estados que forman la UE; en este caso el rumano y el español. Los intentos de aprovechar la nacionalidad de origen han sido especialmente relevantes en el caso del colectivo rumano. Es lo que ocurre con partidos como el Partido Independiente Rumano (PIR) o su variante local en Castellón, el partido Unión Demócrata, así como el PIRUM. Aunque su intención es presentarse en elecciones locales, intentan una vertebración supralocal articulada sobre la presencia de migrantes rumanos en España. 


\section{FUENTES DE DATOS}

En esta investigación se analizan unas organizaciones políticas diseñadas exógenamente, inspiradas y promovidas a partir de la existencia de residentes rumanos en varios municipios españoles. Responden a una lógica electoral de carácter supranacional. Los datos proceden de la investigación documental (medios de comunicación y webs institucionales), análisis estadístico de candidaturas, y entrevistas en profundidad, realizadas entre 2012 y 2016, a representantes de dichos partidos. Una exposición de las técnicas utilizadas en este proyecto para la localización y caracterización de los candidatos, así como su discusión metodológica se puede consultar en Santacreu (2015).

La población rumana en España es muy significativa en términos cuantitativos. En el año de su incorporación a la Unión Europea en 2002, residían en este país casi 670.00 personas de origen rumano. En sólo 6 años la población residente se multiplicó por 10 (720.771 en 2008) -teniendo como hito de referencia el año de incorporación a la UE (2007)- y alcanzando el máximo, en 2012, con casi 800.000 ciudadanos de origen rumano residiendo en España. Indicar que los mayores incrementos se producen en los años de crecimiento de la economía española de los años 2000 y que las cifras se estabilizan, o incluso disminuyen -por razones de retorno- en los años en que la crisis económica ha producido un incremento general de la desocupación. Durante estos últimos años, el descenso de las oportunidades de trabajo para estos trabajadores coincide con el límite máximo temporal para poder percibir las prestaciones sociales por desempleo.

Gráfico 1. Población rumana en España (2002-2016)

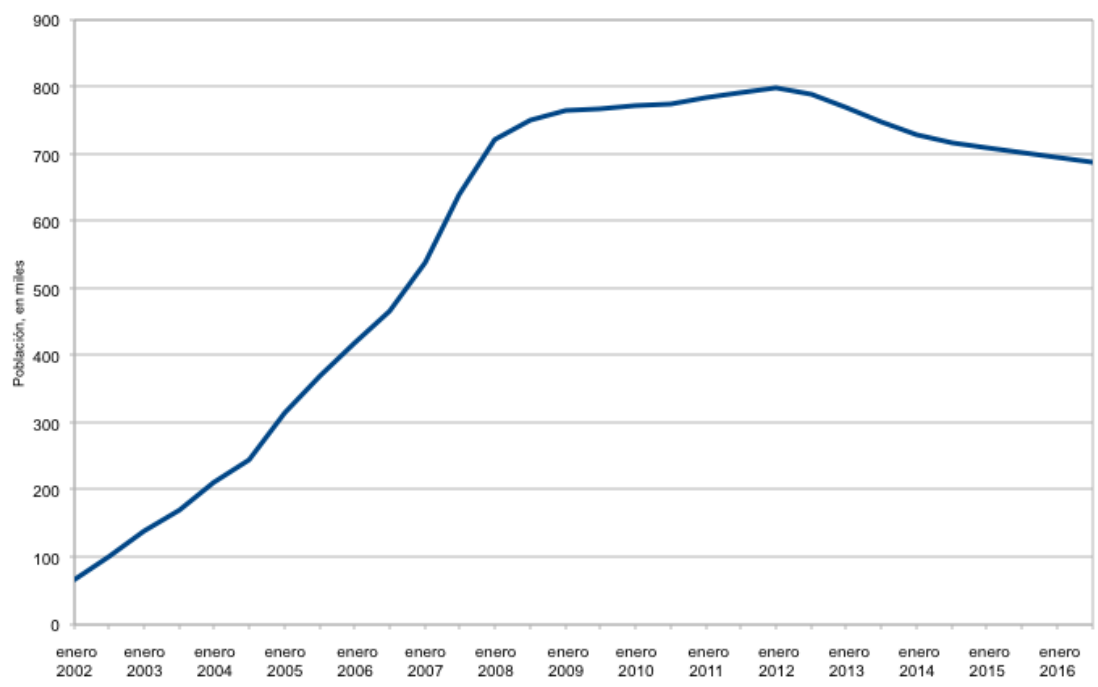

Fuente: elaboración propia a partir de datos del INE (varios años)

En términos electorales, lo más significativo a resaltar es el peso relativo de los rumanos en el municipio, dado que es un indicador de potencial electoral. Se toma la presencia de residentes rumanos como un criterio de referencia de posible apoyo electoral a un partido de carácter étnico-culturalista. Los datos de la tabla 1 corresponden con la densidad de la 
migración rumana en diferentes municipios españoles, en el año en que se ponen en marcha la iniciativa política PIR.

Tabla 1. Población de nacionalidad rumana en porcentaje sobre el total de la población de la localidad en 2007

\begin{tabular}{|l|c|}
\hline Municipio & \% del total \\
\hline Puertomingalvo (Teruel) & $21,6 \%$ \\
\hline Ricla (Zaragoza) & $20,9 \%$ \\
\hline Pomer (Zaragoza) & $19,5 \%$ \\
\hline Estremera (Madrid) & $18,8 \%$ \\
\hline Villamanrique de Tajo (Madrid) & $18,6 \%$ \\
\hline Pradejón (Rioja) & $18,2 \%$ \\
\hline Miralrio (Guadalajara) & $17,6 \%$ \\
\hline Banuelos (Guadalajara) & $17,2 \%$ \\
\hline Carinena (Zaragoza) & $16,7 \%$ \\
\hline Lozoya (Madrid) & $16,6 \%$ \\
\hline Polopos (Granada) & $16,5 \%$ \\
\hline Paniza (Zaragoza) & $15,6 \%$ \\
\hline Arganda del Rey (Madrid) & $15,6 \%$ \\
\hline Tielmes (Madrid) & $15,5 \%$ \\
\hline Condemios de Abajo (Guadalajara) & $15,2 \%$ \\
\hline La Senia (Tarragona) & $14,5 \%$ \\
\hline Carabaña (Madrid) & $14 \%$ \\
\hline Valdilecha (Madrid) & $14 \%$ \\
\hline Villanueva de Alcardete (Toledo) & $14 \%$ \\
\hline Tronchón (Teruel) & $13,9 \%$ \\
\hline Castillejo de Mesleon (Segovia) & $13,7 \%$ \\
\hline Torreblanca (Castellon) & $13,6 \%$ \\
\hline Saceda-Trasierra (Cuenca) & $13,3 \%$ \\
\hline Beniardà (Alicante) & $13,2 \%$ \\
\hline Olmeda de las Fuentes (Madrid) & $12,7 \%$ \\
\hline Calatayud (Zaragoza) & $12,7 \%$ \\
\hline Pozuelo del Rey (Madrid) & $12,7 \%$ \\
\hline Castellón & $12,3 \%$ \\
\hline Alcalá de Henares (Madrid) & $10,1 \%$ \\
\hline Villareal (Castellón) & $10,1 \%$ \\
\hline
\end{tabular}

Fuente: elaboración propia a partir de la Estadística de Padrón Continuo (INE, varios años)

En la mayoría de estos municipios, tanto el PIR (Partido Independiente Rumano) como el PIRUM (Partido Ibérico de los Rumanos) declararon, a través de los medios, estar negociando su implantación y presentación de listas.

\section{LOS PARTIDOS RUMANOS EN ESPAÑA: ENTRE EL TRANSNACIONALISMO Y LA POLÍTICA LOCAL}

En el caso español, los colectivos migrantes de países del Este han mantenido una pauta específica en la creación de partidos propios. Por una parte, los partidos de naturaleza supralocal con aspiraciones de ser el germen de una organización supranacional. En otra, partidos que recurren a la identidad nacional de los migrantes como razón electoral en 
clave local. Ejemplos de esto son, el Partido Ciudadanos Unidos Europeos (PCUE), promovido en 2007 en Gandía por un empresario de la construcción búlgaro que, sin el apoyo de las asociaciones locales, se dirigía preferente a los migrantes rumanos y búlgaros. Y, en otra clave más transparente, el Frente Socialista de Izquierdas que en 2007 se presentó en Puente Genil (Córdoba) con un programa orientado a mejorar las condiciones de vida de los inmigrantes (vivienda, empleo, conciliación, etc.).

Aquí se van a considerar, para el caso español, dos intentos sucesivos de establecer partidos supralocales con aspiraciones trasnacionales apoyados sobre la existencia de un colectivo importante de residentes rumanos.

\subsection{EI Partido Independiente Rumano (PIR)}

El Partido Independiente Rumano (PIR) fue fundado, en 2006 por Augusto Constantín Tecu, un empresario rumano afincado en el municipio castellonense de Burriana. Esta iniciativa contenía un claro objetivo transnacional: vertebrar los colectivos de migrantes rumanos con la finalidad de orientar su participación electoral en las elecciones generales en Rumanía. Este hecho condicionaría al proyecto de partido, tanto en sus definiciones programáticas como en su organización original. Según De la Cal (2006), la fundación del PIR formaba parte de un diseño para constituir un partido paneuropeo de migrantes rumanos. Ese partido, con presencia en diferentes países de la Unión Europea, tendría la utilidad de servir de plataforma de apoyo electoral en Rumania al presidente del partido rumano Nueva Generación, Gigi Becali. Según confirmaba El Periódico de Aragón (2007), Becali estaba impulsando formaciones equivalentes al PIR en otros países europeos. En una entrevista al Periódico Mediterráneo, "Constantin explicó que su formación es 'de centro', pese a compartir 'muchas cosas' con el Partido Nueva Generación, de Rumanía, cuyo cabeza es el multimillonario Gigi Becali (presidente del Steaua de Bucarest). Un partido que, según la presidenta de la Asociación de Inmigrantes de Países del Este, Angela Placsintar, es de marcado carácter "ultranacionalista"' (El Periódico Mediterráneo, 2006).

Al mismo tiempo, junto a la utilidad electoral transnacional, se daba la posibilidad, al menos teóricamente, de adquirir una cuota significativa de poder local. Según De la Cal (2006), las estimaciones de los promotores del partido PIR planteaban que los 381.955 rumanos residentes en España podían llegar a ser en las elecciones municipales de 2007 los votos decisivos para determinar el gobierno local en más de 400 municipios. La base de dicha afirmación era un estudio, entonces en curso, de la Asociación de Emigrantes Rumanos en Castilla-La Mancha. Su presidente, Daniel Comanita, afirmaba que «Son aquellos municipios donde el gobierno local se decidió por un escaso margen de votos en las últimas elecciones municipales de 2003 y ahora cuentan con una significativa comunidad rumana empadronada allí» (De la Cal, 2006). La barrera electoral para acceder al reparto de concejales en las elecciones locales en España es del 5\%, por lo que dada la orientación segmentada de su potencial base electoral, todos los municipios con más del $5 \%$ de censo electoral de población rumana podían ser potencialmente objetivos electorales. Esto ponía en foco municipios de diferentes tamaños poblacionales. De la Cal describe la lógica argumental que seguían los promotores de la iniciativa: "En pueblos como Ricla (Zaragoza) o Pradejón (Madrid) podrían conseguir uno de cada cinco votos. Y sólo en Castilla- León, su participación podría ser decisiva en media docena de municipios con más de 1.000 habitantes, los mismos que en la provincia de Castellón" (De la Cal, 2006). Los municipios en los que se planeaba la expansión del partido venían definidos por la densidad de migrantes rumanos. Esa presencia de migrantes era tomada como referencia de fuerza electoral. Según el Periódico Mediterráneo, "El Partido Independiente Rumano (PIR), constituido en 
Castellón a principios del mes de octubre, calcula que podría obtener al menos siete actas de concejal en los seis municipios de la provincia castellonense en los que pretende presentar candidaturas para las próximas elecciones locales y autonómicas de la primavera del año 2007" (El Periódico Mediterráneo, 2006). La finalidad transnacional se complementaba desde la perspectiva del poder político local, en donde el objetivo era tanto aspirar al gobierno, como ser decisivos en determinadas circunstancias ya que "Su presidente manifestó que, en aquellos casos en los que puedan ser decisivos para la gobernabilidad, apoyarían pactos con PP y con PSPV, según el municipio"(El Periódico Mediterráneo, 2006).

En cierto modo, las dos finalidades, capacidad electoral transnacional y poder político local, se encontraban estrechamente ligadas. Posiblemente, hasta el punto de entorpecerse una a la otra. Es evidente que la lógica de un partido paneuropeo de migrantes rumanos, formado por pequeños partidos en diferentes países, para incrementar la capacidad electoral en Rumania del partido Nueva Generación, casa difícilmente con la lógica de gestión característica de los gobiernos locales. De hecho, algunas de las reivindicaciones se dirigían al ámbito de la política nacional, autonómica o incluso europea, y no a la estrictamente municipal; como es el caso de la reivindicación del acceso a la doble nacionalidad. Otras tenían sentido desde la lógica del fomento de la noción de comunidad e identidad al promover la cultura (bibliotecas), educación (dos idiomas) o religiosa (iglesias ortodoxas). De la Cal (2006) recoge los planteamientos programáticos iniciales de Tecu “¿Para qué dar nuestro voto a unos partidos que no sabemos si nos van a defender realmente cuando podemos conseguirlo nosotros si nos unimos? (...) Nuestras reivindicaciones son muy básicas: queremos bibliotecas, escuelas en dos idiomas, iglesias ortodoxas donde poder ir a rezar -ahora lo hacemos en locales comerciales-, acceso a la doble nacionalidad para poder cobrar pensiones en nuestro país... ¿Nos lo van a dar eso los socialistas o los populares? Ya tenemos 100 afiliados y esperamos conseguir muchos más cuando comience la campaña y nos registremos oficialmente después de la entrada de Rumanía en la UE". Se puede concluir, por tanto, que los objetivos parecen más próximos a una estrategia identitaria nacionalista cultural.

En todo caso, todos estos movimientos organizativos se realizaron en 2006, previamente a la incorporación formal de Rumania a la Unión Europea, prevista para el 1 de enero de 2007; cinco meses antes de las elecciones municipales de mayo de 2007. Según esto, se puede considerar al PIR como un partido de diseño (creado ad hoc con unos fines exclusivamente electoralistas), que realmente no responde a una demanda social concreta por parte de los migrantes rumanos, y que se funda a partir de las nuevas oportunidades políticas que se abren como consecuencia de un cambio institucional. Su estrategia de crecimiento y expansión se apoyaba en las redes informales de los migrantes rumanos, mostrando el carácter "exógeno" del partido. De hecho, su génesis y trayectoria, como veremos más abajo, expresa claramente dicho aspecto. Difícilmente se puede incluir dentro de algún "tipo puro" la modalidad de formación y desarrollo de un partido que nace de una realidad relativamente nueva (transnacionalidad y modificación de los ordenamientos jurídicos políticos a partir de las directrices de un ente supranacional como es la UE). No obstante, siguiendo las discusiones y planteamientos sobre la tipología de partidos (Gunther y Diamond, 2003), podemos incluir estos partidos como "electoralistas" y "étnicos".

La formación de este partido tuvo repercusión en las estrategias y programas de los partidos asentados en la política española. La periodista Lorena Pardo afirmaba que la reacción evidente de los partidos autóctonos fue la coordinación con partidos nacionales de Rumania, como contrapeso a la amenaza electoral del nuevo partido: "En cualquier caso, PP 
y PSOE se han lanzado a la "caza" del voto rumano mediante un acercamiento a aquellas formaciones nacionales del país que propugnan que los inmigrantes rumanos deben decantarse por los partidos españoles como una prueba más de su voluntad de integración. Es el caso del Partido Demócrata Rumano, algunos de cuyos responsables giran visita periódica a la provincia de Castellón como si de una circunscripción rumana más se tratara" (Pardo, 2006). Obsérvese en esta cita la doble incidencia de las redes asociativas transnacionales en ambos sistemas políticos: redefine las estrategias de los partidos del país de destino y amplía la extensión territorial de las campañas electorales en el país de origen: esta actividad de visitar a los residentes en otros países europeos es una práctica extendida de la mayoría de los partidos de los países de origen para, de esta manera, mantener una cuota de electorado emigrante. No es el caso del Reino Unido, donde la emigración y el hecho de fijar la residencia en el extranjero, conlleva la pérdida del derecho al voto en las elecciones nacionales de su país de origen.

Todos estos movimientos del PIR, previamente a su constitución como partido, encontraron un obstáculo legal en la imposibilidad de crear partidos de extranjeros en España. Este impedimento, sumado a su orientación étnica y cerrada e incluso su nombre como partido, tuvo como efecto que, aunque el diseño del partido se anticipara a la integración de Rumania en la UE, su formalización legal entrara en un cierto retraso. Así, El Periódico Mediterráneo (2007) informaba en febrero de 2007 que el PIR "sigue sin estar inscrito en el registro de partidos del Ministerio del Interior, según fuentes de este departamento. La razón, según Tecu, es que se ha decidido cambiar el nombre para retirar "cualquier alusión a Rumanía para hacer ver que nos dirigimos a toda la sociedad". Lorena Pardo, por otro lado, enfatiza la motivación legal en el cambio de nombre, que no de programa "el anunciado PIR, Partido Independiente Rumano, tuvo que cambiar sus siglas por Unión Demócrata Social e integrar a ciudadanos españoles en sus filas para no quebrantar el artículo 6 de la Constitución Española que prohíbe partidos extranjeros” (Pardo, 2007).

Las dificultades que el diseño original encontró, hicieron modificar el nombre del PIR en uno nuevo: la Unión Demócrata Social. En calidad de presidente, Augusto Tecu fue entrevistado poco antes de las elecciones. En sus declaraciones, se aprecia el conflicto que subyace en el cambio de nombre: "Los rumanos deben demostrar a los españoles que tienen también madurez política -dice Augusto-. Los grandes partidos de Castellón han olvidado nuestro potencial. Somos más de 40.000 empadronados". Agustín Tecu se declara socialdemócrata y no quiere confusiones: “¡Este es un partido democrático español, y no rumano! Si no lo escribes así, eres un xenófobo" (Petrescu y Meyer, 2007). Tal y como podría deducirse, estimando exclusivamente sobre la base de los residentes rumanos en los diferentes municipios, las expectativas del partido eran muy elevadas. Como observaba Pardo (2007): "Castellón es la segunda provincia, tras la comunidad uniprovincial Madrid, con más personas de Rumanía para ejercer el derecho al voto. La capital de la Plana registra más de dos mil electores, El municipio de Villarreal supera los 500 y el de Oropesa roza los 300. Según los datos facilitados por la oficina del censo electoral, por municipios, es la ciudad de Castellón la que cuenta con más rumanos inscritos para ejercer el voto el próximo 27 de mayo, con 2.009. Le siguen, Villarreal, con 502; Onda, con 370; Oropesa, con 297; Torreblanca, con 222; Vinaroz, con 201; Vall d'Alba, con 197; Cabanes, con 190; Benicasim, con 150, y Almazora, con 144. En total, de momento, y a falta de la publicación de las listas del censo y la apertura del periodo de reclamación, son un total de 6.673 ciudadanos de Rumanía los que previsiblemente ejercerán su derecho al voto en la provincia de Castellón". 
Sin embargo, en las elecciones de 2007 la Unión Demócrata Social, según datos del Ministerio de Interior (MIR, 2011), solamente presentó lista por Castellón, obteniendo 60 votos y el $0,08 \%$ de los votos válidos. Sin duda, unos resultados muy por debajo de sus expectativas. Las causas para que estos resultados no fueran valorados como buenos por los miembros del partido, son muy variadas; y muchas de ellas ya se apreciaban antes de las elecciones. En especial, las dudas que generaba un partido de estas características. En entrevistas previas a las elecciones, De la Cal (2006), recoge la opinión de varios entrevistados, que en definitiva ilustran lo que es característico de unas elecciones locales: la relevancia del candidato y su imagen, así como las alternativas políticas ya existentes. En ese sentido, la imagen de los políticos en Rumania, en 2007, se encontraba bastante deteriorada entre los migrantes rumanos:

"Yo les votaría si no hubiese partidos generalistas. Pero me fío más de los políticos españoles”, comenta desde El Egido (Almería) Ioana Anastasiu, futura candidata a concejal en las listas del PP en esta localidad (...)

"La gente no se fía de los políticos de su país porque arrastran una gran tradición de corrupción. Los partidos de allí, desde luego, han perdido toda su credibilidad. Se vota según sea de fiable el candidato. Yo creo que un partido rumano en España es una locura porque no ayudaría a nuestra integración, sino todo lo contrario" (De la Cal, 2016).

En las entrevistas en profundidad mantenidas con candidatos rumanos a las elecciones se describe el arraigo de la corrupción y las continuas decepciones experimentadas tras la ilusión que despertaron la caída del régimen comunista en Rumania y las diferentes movilizaciones y protestas que han tenido lugar en este país en el transcurso de las dos décadas posteriores. La última en 2015, que provocó la dimisión del Primer Ministro pero que no condujo a una mejora del sistema político y la administración:

"no lo vi necesariamente como un logro porque el sistema es tan corrupto (...) fue cabeza de turco, esa gente murió porque fue cuestión de licencias ... tú pagas al inspector de turno 20 euros y ya está... desde el más chico hasta el más grande está todo podrido. Pero había hecho mucho por los jubilados, había subido las pensiones, había cumplido parte de las promesas, que ya es mucho. [En aquellas protestas] por una vez vi, por una vez la gente... en Rumanía tú pagas tu Seguridad Social pero una vez que vas al médico le tienes que dar dinero en el bolsillo, para él (...) pero ese tipo de cosas la gente lo acata, no dicen nada. El sistema está podrido y va a hacer falta generaciones... y yo me considero una cobarde porque soy de unas generaciones que no nos deberíamos ir; si nosotros nos vamos quién lo va a arreglar” [EP 17, candidata rumana municipio de Andalucía, 18/07/2016].

El desencanto con la política en Rumanía es una de las causas de la emigración pero, además, influye indirectamente en la participación en las elecciones en España. En ese sentido, la sombra de la política rumana se extiende sobre la imagen de los partidos que toman la idea de Rumanía como referente central en su campaña en España. Así, otro entrevistado duda sobre la pertinencia de su establecimiento en España: "No sé si estamos preparados para tener un partido rumano", dado que los ciudadanos perciben que en su país los partidos: "se mueven por su interés" (El Periódico de Aragón, 2007).

Otro factor de fracaso de un partido supralocal y transnacional, como es este partido, es la naturaleza de las elecciones locales. Estas tienen su propia lógica, ancladas en lo próximo y la persona (Alaminos et. al., 1995), lo que le confiere unas dinámicas 
diferenciadas que matizan las funciones (Sartori, 1980; Von Beyme 1986; Panebianco, 1988) de los partidos en cuanto a reclutamiento, agregación de intereses, formulación de políticas y las propias de la interacción cultural (gestión del conflicto y mediación). En general, esta política local, más pegada a lo cotidiano, hace que los factores ideológicos, partidistas o étnicos tengan menor peso. De hecho, se constata la dificultad de los migrantes de los países del Este entrevistados en este estudio (Rumanos, pero también polacos y búlgaros) para comparar el significado de la dicotomía izquierda-derecha entre España (cuya cultura política tiene un hito no muy lejano como son cuatro décadas de dictadura fascista) y, estos países (excomunistas). El peso que pierde lo ideológico lo gana las características individuales del candidato (imagen, liderazgo, gestión, trato, personalidad) independientemente de su ideología o nacionalidad: "No se puede decir que los electores rumanos y búlgaros sean de derechas o de izquierdas. La situación de allí no coincide con la de aquí. Al votar optan por la persona" (El Periódico de Aragón, 2007). En el mismo artículo un entrevistado rumano destaca que el nuevo partido encontrará dificultades para movilizar el voto migrante a su favor dado que "la gente está contenta con el alcalde".

Al mismo tiempo, la lógica supralocal y étnica del partido entra en una cierta colisión con la integración en la sociedad española. Puede, en ciertas condiciones, percibirse como una amenaza a la sociedad en la que residen. Por ello, como razón de no votar al partido de naturaleza étnica supralocal se encuentra la idea del riesgo de dar apariencia de mostrar ingratitud por el acogimiento; un gesto que puede producir rechazo hacia el colectivo: «Los rumanos no nos vamos a unir en torno a un partido porque no estamos muy organizados. Yo creo que cada uno irá a lo suyo. Además, yo creo que todo el mundo sabe que vivimos en el mejor país del mundo para acoger gente y no van a morder la mano que les da de comer» (De La Cal, 2007).

Este problema es finalmente reconocido por su promotor, Costantin Tecu quien, tras conocer los datos de rumanos inscritos para votar, decide establecer conversaciones con Esquerra Unida y pedir el voto para ellos en Castellón. La razón, "puesto que consideramos que era una lista de inmigrantes para inmigrantes y no nos beneficiaba trabajar solos, y con el acuerdo con EU consideramos que podemos conseguir muchas cosas" (El Periódico Mediterráneo, 2007).

Si los aspectos ideológicos y étnicos no parecen importantes, debemos considerar las condiciones de vida en su país de residencia como argumento esencial de su afinidad partidista, en muchas ocasiones, relacionadas con aspectos tan prácticos como la regulación de la residencia. Como indica un actor rumano afincado en España:

"Los rumanos, como muchos otros emigrantes de los países del Este, tendemos naturalmente hacia el centroderecha por todo lo que sufrimos con la dictadura comunista. Aquí, en cambio, nos ha asustado a veces los discursos xenófobos de algunos dirigentes del PP y otros, en cambio, se quedaron seducidos por el proceso de regularización del PSOE que les ha facilitado mucho la obtención de los papeles" (De la Cal, 2006).

Las conclusiones que cabe extrapolar de estos resultados electorales son que, en primer lugar, parece evidente que el número de residentes rumanos no expresaba realmente su potencial electoral; y, en segundo, que tampoco el voto inscrito rumano se decidió por el voto étnico. Este partido, no obtuvo en la escena política española, la presencia que esperaban conseguir previamente a la celebración de los comicios. A pesar del esfuerzo de este partido por intentar convertirse en el portavoz político de los intereses e inquietudes 
del colectivo rumano residente en España, en las listas de la Unión Demócrata Social sólo se integraba a 17 (un 14,8\%) candidatos del total de candidatos rumanos (115) insertos en el conjunto de listas presentadas en las elecciones locales de 2007 en España. El resto de candidatos de origen rumano se presentaban integrados en otras formaciones.

El partido, como iniciativa supralocal con orientación transnacional, desaparece de la escena política española, no presentándose como tal organización en las siguientes elecciones. Su lugar lo ocuparía otra fuerza política, formada y constituida para las elecciones de 2011: el PIRUM.

\subsection{El Partido Independiente de los Rumanos (PIRUM)}

Si atendemos a su ficha en el registro de partidos políticos en España, el Partido Ibérico de los Rumanos (PIRUM), se inscribe en el registro el 29 de agosto de 2008, con sede social en Sant Celedoni (Barcelona) y actuación de ámbito estatal. Su presentación pública se realizó el 5 de mayo de 2009 en el Convento de los Franciscanos de Barcelona. La idea se gesta desde el Centrul Cultural Român Din Barcelona (Casa România) con una vinculación fuerte a diferentes asociaciones. Su origen en Cataluña es bastante significativo, en el sentido de la referencia a la cultura como elemento de unión entre ciudadanos de diferente nacionalidad. De hecho, en sus comunicaciones hacen referencia a la idea de "fer país" aplicado a la comunidad migrante rumana, si bien con la mirada focalizada en mayor medida en Rumanía que en Cataluña o España. El Movimiento de los Rumanos en Europa parte de una crítica a la situación política en Rumanía, que es diagnosticada como la causa de la apatía de los rumanos emigrados. Es la experiencia en Rumania la que ha convertido a los emigrantes en apáticos y la que ha hecho rechazar la actuación política. En palabras de Florin Bojor (presidente del PIRUM), recogidas en un diario rumano "La palabra política esta maldita para los emigrantes de la diáspora. Por esta razón, nuestras acciones son una mezcla entre cultura, sociedad y política. Me han preguntado miles de veces a qué partido de Rumania pertenecemos" (Rasunetul - Cotidianul bistritenilor de oriunde, 2009). Por ese motivo, por la mala imagen de los partidos en Rumanía, intentar hacer política entre los emigrantes rumanos es "como avanzar en medio de una tormenta de indiferencia y odio".

El nuevo partido reproduce los pasos programáticos y la misma lógica de actuación sobre la base territorial de la concentración de población rumana que caracterizó al PIR, y la posterior Unión Demócrata y Social. El partido afirmaba poseer algo más de un centenar de afiliados, con sedes abiertas en Madrid, Zaragoza, Teruel, Gerona y Alicante. Así mismo, su dinámica de crecimiento y expansión se basa en la idea de redes de contactos interpersonales. Un procedimiento en red que ya empleó anteriormente el PIR como estrategia. La formación y expansión del partido se efectúa mediante una red de contactos personales. Un ejemplo de esta maniobra es la que efectuó Florin Bojor, que entró en contacto con George Fecior, un rumano que residía desde hace diez años en Villamanrique del Tajo y al que animó a formalizar la candidatura del partido en ese municipio. Tal como informa Abenia (2011), el PIRUM aspiraba a presentar listas en una treintena de núcleos urbanos del país. El objetivo era lograr implantarse en localidades con una población rumana elevada, tomando como referencia que este colectivo representara entre el 8 y el $25 \%$ de todos los residentes.

El partido retomó el programa político transnacional y nacional, proponiendo aspectos concretos relacionados con la mejora de la integración de los inmigrantes rumanos, mayor libertad de circulación en la Unión Europea, homologación de los títulos académicos entre Rumanía y España o la construcción de escuelas, centros culturales y bibliotecas bilingües.

El análisis del caso del PIRUM lleva a preguntarse cuáles eran las razones para fundar un partido rumano nuevamente. Es interesante apreciar cómo al transnacionalismo (generar o 
reforzar los vínculos de la comunidad migrante con partidos rumanos de origen) y la reivindicación étnica rumana (cultura), se suma una tercera línea programática, referida a la ideología y vinculada con los movimientos sociales y políticos del país de destino, como es el 15-M. Tal y como expresan en su página web, como declaración de intenciones, el equipo de PIRUM "es conocedor de que no puedes llevarte tu patria contigo, pero si puedes crear una estructura de apoyo inspirada en valores políticos, económicos, culturales...que puedan servir tanto al interés nacional como a los individuos que se encuentran lejos de su patria" (PIRUM, 2011). Respecto a la finalidad del PIRUM, los estatutos del partido expresan ser un "Movimiento de doble objetivo: la preservación de la cultura y lengua de los rumanos que permanezcan en el Oeste y el regreso de los rumanos al país para crear una "Rumania del bien común", bajo el lema catalán "Fer País" construir, cambiar, reformar el país (PIRUM, 2011b).

En lo referido al transnacionalismo, el Presidente del PIRUM afirmaba que "La Europa de la unidad en la diversidad, la Europa de la posmodernidad, si no entiende que debe hacer una identidad europea, un nuevo sistema financiero y una vida de hermandad; repetirá el error de los antepasados romanos de hace 1.600 años, cuando por la falta de identidad, por unas políticas fiscales que han traído la migración de la ciudad a las aldeas y por la falta de fraternidad hicieron que el Imperio Romano desapareciera. Europa está en el mismo camino, si no se despierta Europa desaparecerá...” (PIRUM, 2011a). En definitiva, el diseño del PIRUM alcanza una dimensión trasnacional al apoyarse sobre noción de comunidad de rumanos migrantes, teniendo además una finalidad clara de influir en Rumanía y su política, como muestra las relaciones internacionales y las actuaciones del partido.

La vocación transnacional queda bastante evidente en sus estatutos (PIRUM, 2010a), donde plantea como principio general de su partido "la regeneración de Rumanía y de la mentalidad Rumana; Unidad (Unión de Besarabia con Rumanía) y el regionalismo (descentralización y autofinanciación)". No deja de ser significativo que uno de los principios inspiradores de un partido político en España, de carácter nacional (enfoque supralocal) y base local, tome como referencia la unión de Besarabia (una región del Sureste de la Europa Oriental cuyo territorio está fragmentado por su adscripción a tres países -Moldavia, Rumanía y Ucrania) con Rumanía. De hecho, el movimiento que da luz al PIRUM se diseña con tres ramas: El PIRUM (partido político), la Casa de Rumania en Cataluña (cultural) y un movimiento europeo para estructurar el colectivo rumano migrante en Europa (Movimiento de Rumanos en Europa). Las tres estructuras se definen como una plataforma para incorporar asociaciones, otros partidos, personas, etc. En dicho planteamiento transnacional, se incluyen catorce organizaciones participantes (radicadas en España, Alemania, Italia, Portugal, Austria y Serbia) como fundadoras del Movimiento de los rumanos en Europa (Bojor, s. d.).

En dicha perspectiva transnacional, el PIRUM mantiene una relación conflictiva con varios partidos políticos en Rumanía. Tras ser invitado al Congreso del Partido Social Demócrata, valoró de forma bastante negativa la reunión, concluyendo que el PSD tiene un problema de credibilidad y de ideología (PIRUM, 2010c). Esa dimensión transnacional es la que permite la intervención recíproca desde los partidos rumanos. Así, el presidente del PIRUM critica en la página web del partido (PIRUM, 2010b) las maniobras del Partidului Democrat Liberal (PDL) de Rumanía para boicotear la iniciativa del PIRUM, mencionando a políticos del PDS y PDL rumanos. En su opinión, utilizaron a los presidentes de dos asociaciones del Norte de España, Stela Vidraşcu y Simona Sima, para dividir el Centro Cultural Rumano en Barcelona y así terminar con el PIRUM. Al parecer, al igual que el PIRUM tenía intención programática de intervenir en la política rumana, la política rumana decidió intervenir en las opciones políticas del PIRUM en España. 
En los principios fundacionales de este partido, la cultura rumana se encuentra nuevamente presente en primer plano. Como en el caso de las anteriores formaciones políticas expuestas, se trata de un partido que intenta aprovechar la presencia de un colectivo migrante rumano elevado en España. Intenta utilizar como referencia esencial la identidad de origen, "ser rumano", con el objetivo de dar valor a la masa crítica que la población rumana representa en varios lugares de España. Es, en ese sentido, un intento de base étnica, adoptando las ideas de convivencia y cultura. Sin embargo, es evidente que la referencia étnica no facilita la aceptación por parte de los electores en general, y los promotores confiaban, sobre todo, en la movilización de sus compatriotas para la movilización del electorado.

En los discursos del encuentro para la fundación del partido (Rasunetul Cotidianul bistritenilor de oriunde, 2009), se esbozan ya con claridad estas ideas. En primer lugar, se destaca cómo, a nivel personal, los rumanos se han integrado perfectamente en la sociedad española. La mayoría de ellos tienen en su círculo personal amigos tanto de habla catalana como castellana. Por ello, mejorar la convivencia no es una prioridad. Sin embargo, esta convivencia positiva en el plano individual no se hace extensible a los estereotipos que existen sobre los rumanos como comunidad o nación y por ello se hace una apelación al orgullo nacional. Por ello, la propuesta de Bojor es que si los rumanos se unen en comunidad, serán capaces de cambiar la imagen negativa, los estereotipos y ser respetados como nación. De hecho, la propuesta es crear una conciencia colectiva de regeneración política entre los emigrantes rumanos en Europa, desde la que abordar el cambio del sistema político en Rumanía.

Uno de los elementos más distintivos del PIRUM respecto a su antecesor, el PIR, es un mayor esfuerzo en producir una elaboración ideológica. Según consta en su página web, el "El Partido Ibérico de los Rumanos participará también esta noche 20 de mayo en las manifestaciones de la Plaza Catalunya de Barcelona. Hace dos noches el candidato del PIRUM para Agramunt y el presidente de la rama PIRUM Catalunya, Daniel Dumitru, junto con Florin I. Bojor, el presidente del PIRUM participaron en la manifestación de la Plaza Cataluña de Barcelona contra los partidos "modernos" tradicionalistas, que se olvidaron del mundo, cerrándose en un museo de las ideologías y de las medidas incompatibles con la realidad" (PIRUM, 2011). Según su planteamiento ideológico, el PIRUM no es un partido populista que se une a las reivindicaciones del $15 \mathrm{M}$ para beneficiarse electoralmente, sino que es encuadrable en una nueva categoría: "la posmodernidad". Esta nueva categoría para ubicar ideológicamente a un partido consistiría en que "PIRUM es un partido posmoderno. Las ideologías (derecha, centro, izquierda) después del mayo francés 1968 y de la caída del comunismo en Europa (1989 - 1991) son un discurso anacrónico. La gente no cree más en ideologías y esa cosa lo testifica todo el medio académico. Por tanto, el zoon politikon, como decían los griegos al hombre en su interacción con la sociedad se ha vuelto en una cura para el espacio público en términos de eficiencia, transparencia, pragmatismo, buena gestión, donde la fuerza del ejemplo es el único modelo viable, etc. Cualquier discurso moderno político: ideológico en la Europa del siglo XXI es una reliquia del museo..." (PIRUM, 2011). En ese sentido, el partido se define mediante un concepto, la posmodernidad, y un objetivo, reintegrar a los rumanos al espacio público, tanto si son emigrados como si permanecen en Rumania. Respecto a este objetivo, se articula en esa doble diáspora, geográfica y política de los emigrantes rumanos. En el plano de las ideas políticas, la posmodernidad es definida como la muerte de las ideologías y como propuesta válida para sustituir las ideas por acción. En definitiva, las ideas se constituyen como una herramienta auxiliar de la gestión de un proyecto político concreto. Así, en unas declaraciones, Bojor 
rehuía nuevamente de etiquetas, no queriendo definirse de izquierdas, de derechas o de centro. En especial por el significado de dichas referencias ideológicas en "la memoria reciente de los rumanos, con una dictadura comunista grabada a fuego". Por ello, nuevamente "A su juicio, un partido ha de centrarse en 'la claridad, la eficiencia y la transparencia' en la gestión” (Abenia, 2011).

Ciertamente, cuando la principal característica en común de un electorado es la nacionalidad de origen, el planteamiento de la posmodernidad esquiva las heterogeneidades de origen ideológico que habitualmente segmentan a los votantes. La cuestión central es comprobar hasta qué punto el patriotismo es un elemento movilizador del voto en unas convocatorias electorales locales en el país de residencia al que se ha emigrado. La respuesta es que no ha sido suficiente.

Precisamente, Bojor, haciendo balance de los resultados de las elecciones de 2011 y su decisión de no presentarse a los comicios de 2015, resume con claridad lo que aspiraba a ser el PIRUM: "Todo comenzó como un sueño de fraternidad europea (españoles, catalanes y rumanos), en un contexto de posmodernidad en el que los rumanos aspiran a terminar con los estereotipos negativos sobre ellos (criminales y prostitutas). Una lucha por la igualdad en una Europa unida, una participación democráticamente enriquecedora mediante la participación política en la sociedad Española. La base para que los rumanos regresen a su país para levantar su país. Todo ello define al PIRUM como partido" (Rasunetul - Cotidianul bistritenilor de oriunde, 2015).

Aunque el partido y sus planteamientos están claramente orientados hacia el colectivo rumano, es evidente que el contexto electoral en el que se desenvuelve es el español. La repercusión entre el electorado español es muy semejante a la que se recogía con el PIR. Esencialmente desconfianza y recelo (Europa Press, 2011). Así, el diario $A B C$ en 2011 destacaba en su titular "Desconfianza ante la candidatura del Partido Ibérico de los Rumanos en Villamanrique de Tajo". La clave de la desconfianza procedía precisamente del carácter étnico y cerrado del partido. De tal forma que de todas las promesas electorales pesa especialmente la de que el Partido Ibérico de los Rumanos (PIRUM), un partido político compuesto íntegramente por rumanos, nace con el objetivo de crear 'un futuro mejor' para sus nacionales. Según recoge el periodista en sus entrevistas a ciudadanos del pueblo, "La entrada de un nuevo partido formado exclusivamente por rumanos no ha sido tan bien recibida por parte de los vecinos de Villamanrique de Tajo que creen que si consigue llegar a tener representación en el Ayuntamiento tan sólo velarán por sus intereses" $(A B C, 2011)$. Para ello, cita la opinión de un vecino autóctono, según el cual 'no es normal que se presente una candidatura de rumanos' para formar gobierno en el consistorio: "No somos racistas, estamos bien con ellos, pero que se presente una candidatura no me parece bien" ( $A B C$, 2011). En cierto modo, no se percibe adecuado que un partido político en el ámbito local se dirija de forma explícita a un grupo étnico o migrante concreto. Es una contradicción con la noción de un gobierno local para todos los vecinos. En principio, el partido en el gobierno puede ser uno u otro, pero no se plantea en la práctica que exista una preferencia explícita por unos vecinos respecto a otros.

De hecho, en el corto plazo, la falta de un programa trasversal y la precipitación en la formación de listas y programa les hace ser pesimistas respecto a la intención de entrar a formar parte del pleno del ayuntamiento. No obstante, la apuesta étnica sí les hace ser optimistas a medio plazo: "Una de las candidatas del partido en Villamanrique del Tajo, Lidia Achim, con nueve años de residencia, opinaba que, aunque 'es muy precipitado' llegar a poder conseguir su presencia en el Ayuntamiento, 'dentro de cuatro años por lo menos' podrán contar con la representación de "un concejal"” $(A B C, 2011)$. En definitiva, la clave en 
términos de poder político, como demostrarían otros partidos de residentes extranjeros, es tener el voto decisivo en caso de empate de los partidos mayoritarios. Así, para el caso de Cariñena, Abenia (2011) advierte que "Hasta el propio PIRUM admite que es poco probable que pudieran gobernar en Cariñena. Lo que no quita que obtengan alguno de los 11 puestos de concejal y puedan influir en el color político de quien ostente la alcaldía de un pueblo habituado a las votaciones ajustadas y los pactos entre grupos (los tres ediles del PSOE encabezan la corporación cuando la fuerza más votada fue el PP, con cuatro)". En el caso de Cariñena, la población rumana en 2011 suponía el 22\% del total de habitantes del municipio zaragozano (3.771 empadronados), de los que 686 podrían ejercer su derecho al voto en las elecciones locales. No obstante, Abenia, hacía notar que las expectativas de voto sobre la base del censo de rumanos residentes eran muy relativas. Así, partiendo del referente de las elecciones de 2007, "En los comicios del 22 de mayo podrán participar hasta 50.000 extranjeros en Aragón, cifra que contrasta con los 8.000 que votaron en la cita de 2007" (Abenia, 2011). Ciertamente, se repitió la experiencia pasada con el PIR, según la cual se produjo una diferencia sensible entre el potencial de votantes rumanos y la participación final $\mathrm{y}$, en especial, tomando como elección al partido rumano.

En tanto que partido político, el PIRUM recogía en sus listas al 21,9\% de los candidatos rumanos en las elecciones de 2011 (92 candidatos sobre un total de 421 candidatos). El PIRUM finalmente, formalizaría candidatura en Cariñena, Cartaya, Agramunt, Villamanrique de Tajo y Huelva, muy por debajo de sus expectativas iniciales, que consideraban entre 30 y 50 posibles municipios.

Tabla 2. Resultados del PIRUM elecciones 2011

\begin{tabular}{|c|c|}
\hline & 2011 \\
\hline Cariñena (Zaragoza) & 47 votos $(2,43 \%)$ \\
\hline Cartaya (Huelva) & 17 votos $(0,01 \%)$ \\
\hline Agramunt (Lleida) & 26 votos $(1,08 \%)$ \\
\hline Villamanrique de Tajo (Madrid) & 7 votos $(1,57 \%)$ \\
\hline Huelva & $17(0,01 \%)$ \\
\hline
\end{tabular}

Fuente: elaboración propia a partir de datos del MIR (2011)

Los resultados electorales quedaron muy por debajo de las expectativas. Cosa que ya se predecía en las reflexiones sobre el votante rumano que efectuaban sus líderes. Este hecho tuvo como consecuencia que el PIRUM no se volviese a presentar para las elecciones de 2015. No obstante, dentro del partido se produjo un debate sobre si presentarse en determinados municipios. Finalmente no lo hicieron. Su presidente, Bojor explica por qué: Primero, "porque la comunidad rumana no estaba ni está preparada para ser ciudadanos europeos de primer nivel. Hay casi un millón de rumanos en España que no comprenden que solamente implicándose cívicamente en el proceso electoral pueden llegar a ser importantes. Si solamente votando se es tenido en consideración, no votar lleva a ser considerados ciudadanos de segunda clase. Válidos solamente para trabajar, robar o ser mendigos" (PIRUM, 2015). Segundo, "son pocos los rumanos que comprenden que la integración en España conlleva la aniquilación de la identidad y la cultura rumana. La mayoría de los jóvenes ha adquirido una mentalidad occidental y están orgullosos de comer, beber y vivir en un apartamento hipotecado, con un coche de segunda mano" (PIRUM, 2015). En otras palabras, han olvidado conservar la identidad cultural rumana y no se identifican con el nacionalismo latente de las propuestas del PIRUM. 


\section{CONCLUSIONES}

Las dos experiencias anteriores muestran un intento de combinar la dimensión social y política de lo transnacional. La realidad de una red de emigrantes, con capacidad electoral en su país de origen, que aparece como un potencial de cambio social y político en Rumanía. En ese sentido, se aprecia unas características y dinámicas semejantes en los dos proyectos, así como las contradicciones en las que se ven inmersos. Veamos algunas de las características comunes en las estrategias de los dos partidos y que llevaron al fracaso a ambas organizaciones políticas.

La intención explicita siempre fue movilizar electoralmente el colectivo de migrantes rumanos, con un enfoque esencialmente étnico. Claramente eran partidos pensados para dar voz y capacidad de influencia política a los residentes europeos rumanos, supuestamente en dos ámbitos de difícil encaje: su país de origen y los municipios de residencia. Esa estrategia impidió que lograran consolidarse y presentar listas en todos los municipios a los que aspiraban, dado que el único elemento en común era su nacionalidad de origen. Así mismo, al ser su objetivo la población rumana, se limitaba drásticamente su potencial al colectivo migrante.

Lo anterior se refleja en que las propuestas electorales, producían una elevada segmentación de los electorados locales, dado que por un lado se centraban en modo general en la mejora de vida y de la imagen de los residentes rumanos. Por otro, en la defensa de la identidad rumana y la renovación democrática de su país de origen. Promover simultáneamente la diferenciación (cultural) y la integración (social) es una tarea difícil de comunicar.

En ese sentido, la aspiración de acción transnacional se concreta en ambos partidos en dos líneas de acción. La primera es apelar a la cultura como elemento identitario y, la segunda, mantener una presencia política en Rumanía mediante el contacto con los partidos rumanos. La primera línea de acción hace énfasis en la dimensión cultural. Promover el nacionalismo cultural, como parte esencial de la definición identitaria de su electorado subyace a la formación de estos partidos. Es una tarea simultánea, crear una imagen de comunidad entre los migrantes rumanos tomando como base política sus culturas y tradiciones. Nuevamente, este enfoque reaparecerá en el PIRUM, inspirándose en el nacionalismo catalán, y al que harán mención expresa con la idea de "Fer País". En este caso, crear una comunidad.

La segunda línea de acción transnacional se concreta en que los partidos locales dirigidos a residentes rumanos en España no dejan de atender y estar presentes, tanto mediáticamente como políticamente, en Rumanía. Así, la fundación del PIR recibió cobertura mediática en este país, manteniendo contactos con el partido Nueva Rumania, en una clara intención transnacional, que más tarde el PIRUM mantendría como parte de su agenda.

La estrategia para llegar a sus votantes se basa en la lógica de redes, mediante el proselitismo entre los compatriotas radicados en los municipios. Esa estrategia es iniciada por el PIR y reproducida por el PIRUM, empleando las redes de contactos y asociaciones para intentar extender y consolidar al partido. Las redes son una forma específica de llegar a los votantes, por la que los electorados no se estructuran geográficamente o ideológicamente, sino mediante redes de contactos (amigos, vecinos, asociados, centros culturales, etc.). La densidad de la red y la característica que defina la relación condiciona, sin duda, el éxito de este tipo de campañas.

Por otro lado, sus cálculos electorales eran muy simples y se apoyaban sobre las estimaciones de votante rumano. Mayor presencia de residentes rumanos supone mayor posibilidad de obtener buenos resultados. No obstante, si comparamos la población que 
define el potencial electoral desde el punto de vista transnacional en el caso rumano, con la inscripción final para poder votar y los resultados electorales, destacan dos ideas. La primera, que existe un diferencial notable entre el voto potencial y los que realmente se inscriben. Segundo, que el voto rumano inscrito no se dirigió a los partidos que se definían como rumanos. Por el contrario, cabe apreciar una elección preferente entre las candidaturas de partidos autóctonos.

Se trata de dos cuestiones ligadas y condicionadas formalmente por la necesidad de inscripción previa. La primera es movilizar el voto y la segunda convencerle de que vote al partido en concreto. En las dos fases los partidos trasnacionales no tuvieron éxito.

Una de las cuestiones evidentes es que una cosa es la existencia de una masa de residentes rumanos, y otra su movilización electoral. El ser rumano, per se, no parece un argumento movilizador. De hecho, implica una referencia cultural-nacionalista que puede estar alejada de las expectativas de los residentes rumanos. Este colectivo, por lo general, es bastante poco participativo tanto social como políticamente, y la estrategia de integración les es más atractiva que la de diferenciación que anima al PIR.

Al mismo tiempo, la posibilidad de confundir códigos "políticos” es mínima. Un residente rumano puede tener alguna confusión sobre la lectura ideológica de los partidos españoles, especialmente si es recién llegado, pero tiene una idea muy clara del significado de los partidos, códigos, símbolos y expresiones de los partidos y políticos rumanos. En ese sentido, se puede producir un alejamiento ideológico de partidos culturalistas que, aunque se proponen de centro, no reflejan la gestión como su principal característica, y establecen afinidades con partidos rumanos. En los dos partidos (PIR y PIRUM) no es la gestión su principal argumento, sino unas referencias claramente nacionalistas. Eso le aleja de la lógica propia de los partidos locales, llevando a primer plano una lectura en clave de política rumana, con la consecuente desconfianza.

Finalmente, una característica distintiva del PIRUM es su intento de vincularse a la crítica a los partidos políticos tradicionales que se produce en España en 2011. En ese sentido, aspira a traducir los movimientos de protesta que surgen en España en términos de la política en Rumanía. Produce con ello una adenda ideológica que propone una nueva política "posmoderna", abandonando las categorías de derecha e izquierda como claves de lectura ideológica.

En resumen, los intentos de establecer el germen de partidos transnacionales encuentran varias contradicciones importantes en clave electoral. Por una parte, la lógica local de las elecciones choca con su intención de vertebración supralocal y transnacional. Existe una evidente dificultad en elaborar un programa común que se aproxime a la realidad local que se debate en dicho tipo de elecciones. Otra contradicción evidente es la defensa de la renovación democrática en Rumanía mediante candidaturas locales en España. Por último, la base étnica de su electorado condiciona el alcance de sus programas electorales y limita drásticamente su base electoral.

\section{BIBLIOGRAFÍA}

ABC (2011) "Desconfianza ante la candidatura del Partido Ibérico de los Rumanos", en https://goo.gl/rBDMn9 [Consulta 15/02/2017]

Abenia, E. (2011) "Un partido rumano se presentará por primera vez a las municipales de mayo", Heraldo 08/01/2011, en https://goo.gl/Gxw3Gv [Consulta 01/12/2016]

Alaminos, A. et. al. (1995) Gestión de la vida local. Alicante: Diputación de Alicante. 
Alaminos, A.; Penalva, C.; Perea, N. (2016) "Si usted fuera migrante. Las preferencias de candidato como hipótesis". Sociologiados. Revista de investigación social, 1(2): 51-68.

Alaminos, A.; Penalva, C.; Perea, N. (2017) "La opinión pública europea y el derecho al voto de los migrantes. Sistema (en prensa).

Basch, L.; Schiller, N. G. y Szanton-Blanc, C. (1994) Nations Unbound: Transnational Projects, Postcolonial Predicaments and Deterritorialized Nation-State. New York: Gordon \& Breach.

Bojor, P. (s. d.), "Mişcarea românilor din Europa", http://www.larg.de/?archive\&id=194 [Consulta 17/02/2017]

Boli, J., Thomas, G. M. (1999) Constructing world culture: International nongovernmental organizations since 1875, Stanford, Calif: Stanford University Press.

Coutin, S. B. (2000) "Denationalization, Inclusion, and Exclusion: Negotiating the Boundaries of Belonging". Indiana Journal of Global Legal Studies 7 (2): 585-93.

De la Cal, J. C. (2006) "Los rumanos ya tienen su partido en España". Crónica (suplemento del diario El Mundo), en https://goo.gl/vp8UfZ [consulta 10/01/2017]

El Periódico de Aragón (2007) "El Partido Independiente Rumano quiere presentar lista en Zaragoza", en https://goo.gl/dj7EeS [consulta 10/01/2017]

El Periódico Mediterráneo (2006) "El partido rumano tendrá lista en seis municipios", en https://goo.gl/X1ZD92 [consulta 10/02/2017]

El Periódico Mediterráneo (2007) "El partido de los rumanos cambia de nombre y sigue sin inscribirse en el registro de Interior" en https://goo.gl/FuhcGa [consulta 10/01/2017]

Europa Press (2011, abril 26) PIRUM, partido candidato en Villamanrique de Tajo [Archivo de video]. Recuperado de https://www.youtube.com/watch? $\mathrm{v}=\mathrm{RPeBEtMmhag} \&$ feature=youtu.be

Glick-Schiller, N.; Basch, L. y Szanton-Blanc, C. (1992) "Towards a Definition of Transnationalism. Introductory Remarks and Research Questions". En N. Glick-Schiller, L. Basch y C. Szanton-Blanc (Eds.) Towards a Transnational Perspective on Migration: Race, Class, Ethnicity and Nationalism Reconsidered, New York: New York Academy of Sciences, pp. IX-XIV

Glick-Schiller, N. y Fouron, G.E. (1999) "Terrains of Blood and Nation: Haitian Transnational Social Fields". Ethnic and Racial Studies, 22(2): 340-366.

Guarnizo, L.E.; Portes, A. y Haller, W. (2003) "Assimilation and Transnationalism: Determinants of Transnational Political Action among Contemporary Migrants", American Journal of Sociology, 108 (6).

Gunther, R. y Diamond, L. (2003) Species of Political Parties. A new typology. Party Politics, 8 (2): 167-199.

INE (varios años) Estadística de Padrón Continuo. Madrid: Instituto Nacional de Estadística, en https://goo.gl/LAfgKi

Keck, M.E. y Sikkink, K. (1998) Activists beyond Borders: Advocacy Networks in International Politics, Ithaca, N.Y.: Cornell University Press.

Lafleur, J.M. (2012) "Transnacionalismo, diáspora y voto en el exterior". En Lafleur, J.M. (Ed.), Diáspora y voto en el exterior, Barcelona: CIDOB.

Levitt, P. y Schiller, N.G. (2004) "Conceptualizing Simultaneity: A Transnational Social Field Perspective on Society". International Migration Review, 38 (3).

Malamud, A. (sin fecha), Partidos Políticos en la Unión Europea: una contradicción en los términos. goo.gl/I3Zsia [Consulta 03/03/2017].

MIR (2011). Elecciones locales 2011, goo.gl/6nS6M7 [Consulta 25/11/2016].

Moldes, R. (2015) "De la "integración" a la "conectividad": expectativas laborales y formas de participación de la emigración cualificada", Arxius de Ciències Socials, 33: 127-140.

Montero, J. R. y Gunther, R. (2004) "Los estudios sobre los partidos políticos: una revisión crítica". Postdata, 10. Versión on-line (goo.gl/7GQ8jD) [Consulta 1/02/2017]

Panebianco, A. (1988) Political Parties: Organization and Power, Cambridge: Cambridge University Press.

Pardo, L. (2006) "Crean en Castellón el primer partido político de rumanos". $A B C$ 02/11/2006, goo.gl/uNr2sF [Consulta 10/02/2017] 
Pardo L. (2007) "Casi siete mil rumanos han solicitado ya su derecho a votar en Castellón". $A B C$ 17/04/2007. goo.gl/gjaLIy [Consulta 10/01/2017]

Parra, J.F. (2016) "Acercamiento al derecho de la migración y la ciudadanía transnacional. El caso de los emigrantes mexicanos y sus derechos políticos", América Latina Hoy, 33: 73-100.

Petrescu, A. y Meyer M. (2007) "Candidato turista, candidato inmigrante". Interviu 21/05/2007. http://www.interviu.es/reportajes/articulos/candidato-turista-candidato-inmigrante\# [Consulta $12 / 12 / 2016]$

PIRUM (2010a) "Constituirea Publică a Partidului Românilor din Spania”, goo.gl/r9W7sF [Consulta $15 / 02 / 2007]$

PIRUM (2010b) "Pedeliştii vor să spargă Partidul Românilor din Spania, PIRUM", 12/10/2010 goo.gl/85mqb9 [Consulta 15/2/2017]

PIRUM (2010c) "Congresul PSD: bâlci de interese”, 12/10/2010 https://goo.gl/9ZOCJI [Consulta $13 / 01 / 2017]$

PIRUM (2011a) "PIRUM participa en las manifestaciones de Barcelona", 20/05/2011 https://goo.gl/1Vjx4W [Consulta 13/01/2017]

PIRUM (2011b) “Statut", https://pirum.wordpress.com/statut/ [Consulta 13/01/2017]

PIRUM (2015) "De ce românii din Spania nu candidează la alegerile locale din 2015?". 10/03/2015, https://goo.gl/i1ObLS [Consulta 13/01/2017]

Portes, A. (1996) "Transnational Communities: Their Emergence and Significance in the Contemporary World System". En , Roberto Patricio Korzeniewicz y William C. Smith (Eds.) Latin America in the World Economy. Westport, Conn.: Greenwood Press.

Portes, A. (2001) "Debates y significación del transnacionalismo de los inmigrantes" en Revista de Estudios Migratorios Latinoamericanos, 49: 469- 484.

Portes, A. (2003) "Conclusión: Hacia un nuevo mundo. Los Orígenes y efectos de las actividades transnacionales". En A. Portes et. al. (Eds), La globalización desde abajo: transnacionalismo inmigrante y desarrollo, la experiencia de Estados Unidos y América Latina, México: Facultad Latinoamericana de Ciencias Sociales Sede-México, pp. 377-395.

Portes, A. (2005) "Convergencias teóricas y evidencias empíricas en el estudio del transnacionalismo de los inmigrantes". Migración y Desarrollo, 4: 2-19.

Portes, A.; Guarnizo, L. E. y Landolt, P. (1999) "The Study of Transnationalism: Pitfalls and Promise of an Emergent Research Field". Ethnic and Racial Studies, 22 (2).

Portes, A.; Escobar, C. y Arana, R. (2008) "¿Lealtades divididas o convergentes? Informe sobre la incorporación política de inmigrantes latinoamericanos en los Estados Unidos". En Nuevos Retos del Transnacionalismo en el estudio de las migraciones. Madrid: Ministerio de Trabajo e Inmigración.

Pries, L. (2001) "The disruption of social and geographic space mexican-us migration and the emergence of transnational social spaces", International Sociology, 16 (1): 55-74.

Rasunetul - Cotidianul bistritenilor de oriunde (2009) "S-a constituit Partidul Iberic al Românilor din Spania“, 05/08/2009 (Melania Cuc), goo.gl/QXKVH5 [Consulta 15/02/2017]

Rasunetul - Cotidianul bistritenilor de oriunde (2015) "Florin Bojor: De ce românii din Spania nu candidează la alegerile locale din 2015?”, goo.gl/oqS9De [Consulta 15/02/2017]

Reglamento CE 2004/2003 del Parlamento Europeo y del Consejo de 4 de noviembre de 2003, relativo al estatuto y la financiación de los partidos políticos a escala europea. DO L 297 de 15.11.2003.

Rocco, R. (2006) "Transforming Citizenship: Membership, Strategies of Containment, and the Public Sphere in Latino Communities." En S. Oboler (Ed.) Latinos and Citizenship: The Dilemma of Belonging. New York: Palgrave Press, pp. 301-328.

Santacreu, O. (2005) "Muestreo de candidatos extranjeros en las elecciones locales españolas. Nota metodológica". Obets. Revista de Ciencias Sociales. 10 (2): 473-489.

Sartori, G. (1980) Partidos y sistemas de partidos. Madrid; Alianza Editorial.

Sassen, S. (2002) "The Repositioning of Citizenship: Emergent Subjects and Spaces". Berkeley Journal of Sociology, 46: 4-24. 
Sentencia 48/2003 del Tribunal Constitucional. Recurso de inconstitucionalidad núm. 5550-2002. Promovido por el Gobierno Vasco contra diversos preceptos de la Ley Orgánica 6/2002, de 27 de junio, de partidos políticos. BOE núm. 63, de 14 de marzo.

Smith, J.; Chatfield, C. y Pagnucco, R. (Eds.) (1997) Transnational Social Movements and Global Politics: Solidarity beyond the State, Syracuse, NY.; Syracuse University Press.

Smith, P.M. y Guarnizo, L.E. (Eds.) (1998) Transnationalism from Below. NJ: Transaction Publishers (col. Comparative Urban and Community Research, 6).

Tarrow, S. (1998) Power in Movement: Social Movements and Contentious Politics, Cambridge: Cambridge University Press.

Vertovec, S. (2003) "Migrant Transnationalism and Modes of Transformation", International Migration Review, 38: 970-1001.

Vertovec, S. (2007) "Super-diversity and its implications", Ethnic and racial studies, 30 (6): 10241054.

Von Beyme, K. (1986) Los partidos políticos en las democracias occidentales. Madrid: CIS.

\section{Breve currículo:}

\section{Antonio Alaminos Chica}

Catedrático de la Universidad de Alicante en el departamento de Sociología II e investigador del Instituto Interuniversitario de Desarrollo Social y Paz. Sus líneas de investigación se orientan al estudio de los movimientos sociales, la participación política y la movilidad intraeuropa, las prácticas económicas de resiliencia, así como las mediciones alternativas al desarrollo. Publicaciones recientes, en colaboración con otros autores: "Cuando despertaron, la democracia todavía estaba allí" en Cultura, Lenguaje y Representación; "If you were a migrant. Candidate preference as a hypothesis" en Sociologiados; "Visions of the Spanish Revolution: identities and conflicts in post-welfare societies", en Convergencia. Revista de Ciencias Sociales; "Forecasting elections with high volatility" en Italian Journal of Applied Statistics; "Resilience by solidary ties" en Obets. Revista de Ciencias Sociales.

\section{Clemente Penalva Verdú}

Profesor Titular de la Universidad de Alicante en el departamento de Sociología II e investigador del Instituto Interuniversitario de Desarrollo Social y Paz. Sus líneas de investigación se orientan al estudio de los movimientos sociales, la participación política y la movilidad intraeuropa, las prácticas económicas de resiliencia, así como las mediciones alternativas al desarrollo. Publicaciones recientes, en colaboración con otros autores: "Reflections and challenges of international social work research" en Critical and radical social work; "Visions of the Spanish Revolution: identities and conflicts in postwelfare societies", en Convergencia. Revista de Ciencias Sociales; "La protesta en España y Brasil: crisis económica y crisis política" en Obets; y "Antiausteridad y protesta en el contexto de la crisis económica y política en España e Italia” en Società Mutamento Politica. 